\title{
Weak measurements of trajectories in quantum systems: classical, Bohmian and sum over paths
}

\author{
A. Matzkin \\ Laboratoire de Physique Théorique et Modélisation (CNRS Unité 8089), \\ Université de Cergy-Pontoise, 95302 Cergy-Pontoise cedex, France
}

\begin{abstract}
Weak values, obtained from weak measurements, attempt to describe the properties of a quantum system as it evolves from an initial to a final state, without practically altering this evolution. Trajectories can be defined from weak measurements of the position, or inferred from weak values of the momentum operator. The former can be connected to the asymptotic form of the Feynman propagator and the latter to Bohmian trajectories. Employing a time-dependent oscillator as a model, this work analyzes to what extent weak measurements can shed light on the underlying dynamics of a quantum system expressed in terms of trajectories, in particular by comparing the two approaches.

PACS numbers: 03.65.Ta, 03.65.Ca
\end{abstract}




\section{INTRODUCTION}

Contrary to classical physics, the standard formalism of quantum mechanics forbids the use of space-time trajectories to describe the time evolution of a system. However trajectories surreptitiously sneak back into the description, the interpretation and the computation of quantum phenomena. There are various form of trajectories that have been found useful. Among these the most prominent are the paths of the path integral approach due to Feynman [1] and the trajectories built on the probability flow employed in the de Broglie-Bohm model [2]. Both types of trajectories have been employed to interpret experimental results.

The path integral approach has been extremely successful for quantum systems in the semiclassical regime. Indeed, in this regime the path integral becomes essentially a coherent sum over the classical trajectories of the corresponding classical system [1]. Such classical trajectories have been employed to understand the properties of these systems in the framework of "quantum chaos" [3], and their manifestations have have been experimentally observed in many quantum systems (see eg Refs [4]). The trajectories of the Bohmian model are essentially obtained by following the probability current density arising from the Schrödinger equation. Bohmian trajectories have also been employed to interpret the dynamics in several systems [5]. They have further been used as a numerical computation method, especially in molecular physics or when mixing classical and quantum degrees of freedom in a mean field approximation is required [6].

The trajectories of the path integral, generated by the classical Lagrangian, are generically different from the quantum trajectories built on the Schrödinger probability flow [7]. This is not a problem as long as one sees these trajectories as being computational tools or mathematical artefacts. However recently the approach of weak measurements, introduced some time ago by Aharonov, Albert and Vaidman [8] has been employed to measure non-perturbatively trajectories in quantum systems. The main idea underlying weak measurements [9-11] is to access the properties of a quantum system evolving from a given initial state towards a final state, practically without disturbing the system evolution. This cannot be achieved by a standard projective measurement (doing so would irremediably disturb the system by projective its premeasurement state to a subspace spanned by the eigenstate of the measured observable). Instead, the system observable is coupled unitarily to an ancilla acting as a weak measurement apparatus (WMA). If the coupling is weak, the unitary evo- 
lution of the system is practically left unperturbed. The value recorded by a WMA is not an eigenvalue (since there is no state projection) but what is known as a weak value [8] of the weakly measured observable.

The idea of inferring Bohmian trajectories from weak measurements of the momentum (followed by a projective measurement of the position) has been proposed first by Leavens [12] (see also [13 15] for more recent approaches). This scheme was experimentally implemented in a two-slit interferometer [16] allowing to reconstruct Bohmian trajectories from the observed data. A method that can in principle allow to observe the Feynman paths with weak measurements of the position (including the coherent paths superposition) was also suggested recently [17] (see also [18]). In a first view it is therefore tempting to conclude that the type of trajectory that one sees depends eventually on what is being measured, which in turn calls for a definite experimental setup.

The aim of this work is to examine this question in details by displaying expressions for the weak measurement of classical and Bohmian trajectories in the same system. We will employ a tractable model system - a two dimensional time-dependent linear oscillator (TDLO). While the dynamics of the TDLO is arguably less rich than that of generic systems, its main advantage in the present context is that the Feynman sum over paths can be obtained exactly in closed form (without invoking the semiclassical approximation) while the computation of the Bohmian trajectories is numerically tractable. At the same time the time-dependent aspect allows to "simulate" dynamical feature that generally appear in systems with more involved dynamics (like recurrences of closed orbits). Moreover the TDLO has often been employed to model quantum systems such as the dynamics of trapped ions [19], photon generation in quantum optics [20] or cosmological mini-superspace models [21].

The paper is organized as follows. We first introduce the weak measurements framework and derive the two types of trajectories that can be inferred from weak measurements. We then introduce our model system and detail how the quantum dynamics can be interpreted in terms of classical or Bohmian trajectories (Sec. III), yielding different interpretations of dynamical phenomena when classical and Bohmian trajectories differ. Sec. IV describes the trajectories inferred from weak measurements for the TDLO, including derivations and several numerical illustrations for specific cases. We discuss our findings and conclude in Sec. V, while an Appendix details how we obtain the closed form solutions of the Schrödinger 
equation for the TDLO.

\section{WEAK MEASUREMENTS}

\section{A. Weak measurement framework}

The underlying idea at the basis of the weak measurement framework is an attempt to answer the question:" what is the value of a property (represented by an observable $\hat{A}$ ) of a quantum system while it is evolving from an initial state $\left|\psi\left(t_{0}\right)\right\rangle$ to a final state $|\chi\rangle$ ?". Instead of making a projective measurement the observable $\hat{A}$ is coupled unitarily to a dynamical variable of an ancilla (the weak measurement apparatus, WMA). There is thus no projection of the system's quantum state at this stage. Moreover if the coupling is asymptotically weak, it can be shown that the state of the system is left practically undisturbed. The system thus continues ${ }^{1}$ its evolution until a final projective measurement (of another observable $\hat{B})$ projects its state to $|\chi\rangle$, the post-selected state. As a result of the unitary coupling, a projective measurement on the system also modifies the quantum state of the WMA: the variable conjugate to the coupled one is shifted by a quantity proportional to $\operatorname{Re}\left\langle A_{w}\right\rangle$ where $\left\langle A_{w}\right\rangle$ is the weak value of the observable $\hat{A}$ when the system is pre-selected in state $\left|\psi\left(t_{0}\right)\right\rangle$ and post-selected to the state $|\chi\rangle$. Letting $U\left(t^{\prime \prime}, t^{\prime}\right)$ denote the evolution operator of the system between times $t^{\prime}$ and $t^{\prime \prime},\left\langle\hat{A}_{w}\right\rangle$ is given by

$$
\left\langle\hat{A}_{w}\right\rangle=\frac{\langle\chi| U\left(t_{f}, t_{w}\right) \hat{A}\left[U\left(t_{w}, t_{0}\right)\left|\psi\left(t_{0}\right)\right\rangle\right]}{\left\langle\chi\left|U\left(t_{f}, t_{0}\right)\right| \psi\left(t_{0}\right)\right\rangle}
$$

where $t_{w}$ and $t_{f}$ stand for the times at which the weak measurement and final postselection take place respectively. Introducing the notation $\left\langle\chi\left(t_{w}\right)\right| \equiv\langle\chi| U\left(t_{f}, t_{w}\right)$ representing the postselected state evolved backward in time, while $\left|\psi\left(t_{w}\right)\right\rangle=U\left(t_{w}, t_{0}\right)\left|\psi\left(t_{0}\right)\right\rangle$, the weak value (1) is written in terms of quantities taken at the weak measurement time $t_{w}$ as

$$
\left\langle\hat{A}_{w}\right\rangle=\frac{\left\langle\chi\left(t_{w}\right)|\hat{A}| \psi\left(t_{w}\right)\right\rangle}{\left\langle\chi\left(t_{w}\right) \mid \psi\left(t_{w}\right)\right\rangle} .
$$

\footnotetext{
${ }^{1}$ Formally, the system and the WMA get entangled, so the coupling interaction changes the dynamics of the overall entangled wavefunction. For a small coupling, the postselection probabilities are not modified to first order while the WMA's wavefunction picks up a shift; see eg Secs 2 and 5 of [9], and [22] for a problem solved with exact solutions of the Schrödinger equations.
} 
Hence the WMA acts as a pointer that records the weak value ${ }^{2}$ of the weakly measured observable. While there has been a controversy on the meaning of weak values from their inception, in our view the controversy has more to do with the interpretation given to the theoretical terms of quantum theory in general than to the specificities of weak measurements as such. There is now ample evidence [9, 10] that the weak values given by Eq. (1) capture a universal effect in which $\operatorname{Re}\left\langle A_{w}\right\rangle$ represents the response on the probe of a minimally disturbing interaction for the system reflecting the value of the property described by $\hat{A}$ relative to the fraction of the initial state that will conditionally end up in the post-selected state $|\chi\rangle$. The basic property allowing this interpretation follows by writing the expectation value of $\hat{A}$ when the system is in the state $\left|\psi\left(t_{w}\right)\right\rangle$ in terms of the probabilities of reaching each eigenstate $\left|\chi_{f}\right\rangle$ of a different observable $\hat{B}$,

$$
\left\langle\psi\left(t_{w}\right)|\hat{A}| \psi\left(t_{w}\right)\right\rangle=\sum_{f}\left|\left\langle\chi_{f} \mid \psi\left(t_{w}\right)\right\rangle\right|^{2} \operatorname{Re} \frac{\left\langle\chi_{f}|\hat{A}| \psi\left(t_{w}\right)\right\rangle}{\left\langle\chi_{f} \mid \psi\left(t_{w}\right)\right\rangle},
$$

where the restriction to the real part comes from the fact that since the left-handside is real, the sum over the imaginary parts vanishes. The interpretation of this formula in terms of weak measurements assumes impilictly that the probabilities of obtaining the final state $\left|\chi_{f}\right\rangle$ are not modified by the action of $\hat{A}$.

Note that in terms of projective measurements, Eq. (3) can also be read similarly to the standard quantum mechanical expectation value expression

$$
\left\langle\psi\left(t_{w}\right)|\hat{A}| \psi\left(t_{w}\right)\right\rangle=\sum_{f}\left|\left\langle\alpha_{f} \mid \psi\left(t_{w}\right)\right\rangle\right|^{2} \alpha_{f}
$$

where $\hat{A}\left|\alpha_{f}\right\rangle=\alpha_{f}\left|\alpha_{f}\right\rangle$. While Eq. (4) involves a protocol in which the expectation value is obtained by measuring the eigenvalues $\alpha_{f}$ of $\hat{A}$ and their relative frequencies, Eq. (3) suggests a protocol in which the expectation value of $\hat{A}$ is obtained by a weak measurement of $\hat{A}$ followed by a standard projective measurement of $\hat{B}$, for which only the relative frequencies for obtaining the eigenvalues $\chi_{f}$ of $\hat{B}$ are needed.

\footnotetext{
${ }^{2}$ The pointer can actually register either the real or the imaginary parts of the weak value, which is a complex number. Only the real part is related to the value of the measured observable, while the imaginary part is typically related to the measurement backaction [23].
} 


\section{B. Weak measurements of position: "weak" trajectories}

The most intuitive way of measuring a trajectory follows from its definition: in a given frame of reference the position $\mathbf{r}(t)$ is recorded as a function of time. For an evolving quantum system, this involves monitoring the position not only non-destructively, but without affecting the subsequent evolution of the system. A weak measurement of the position is perfectly suited in order to monitor the position. Starting from a localized initial state $\left|\psi\left(t_{0}\right)\right\rangle$ - this will be the preselected state -, and ending with a projective measurement to a state localized at a given final position at time $t_{f}$ (this will be the postselected state), we can place a series of weak measurement apparata (WMA) that weakly interact with the system via a local coupling to the position observable $\hat{\mathbf{r}}$.

Assume first that there is a single WMA, lying at position $\mathbf{R}^{0}$ and whose wavefunction $\phi(\mathbf{R})$ is tightly localized around the central position $\mathbf{R}^{0}$, eg a Gaussian wavefunction $\phi(\mathbf{R})=$ $\left(2 / \pi \Delta^{2}\right)^{1 / 2} e^{-\left(\mathbf{R}-\mathbf{R}^{0}\right)^{2} / \Delta^{2}}$. The weak interaction is triggered when the system wavefunction enters the region around $\mathbf{R}^{0}$, corresponding to a contact interaction of the form

$$
H^{0}=\gamma(t) \hat{\mathbf{r}} \cdot \mathbf{R}^{0} f\left(\left|\hat{\mathbf{r}}-\mathbf{R}^{0}\right|^{2}\right)
$$

where $\gamma$ is a smooth function of $t$ determining the coupling and $f$ is a function sharply peaked at $\left|\hat{\mathbf{r}}-\mathbf{R}^{0}\right|=0$ indicating the short-range character of the contact interaction between the system and the WMA. Let $t_{w}$ denote the mean time at which the interaction takes place. If the duration $\tau$ of the measurement is short relative to the timescale of the system dynamics, then [24] the WMA records ${ }^{3}$ the weak value of the position at time $t_{w}$

$$
\left\langle\mathbf{r}\left(t_{w}\right)\right\rangle_{W} \equiv \frac{\left\langle\chi\left(t_{w}\right)\left|\hat{\mathbf{r}} f\left(\left|\hat{\mathbf{r}}-\mathbf{R}^{0}\right|^{2}\right)\right| \psi\left(t_{w}\right)\right\rangle}{\left\langle\chi\left(t_{w}\right) \mid \psi\left(t_{w}\right)\right\rangle} .
$$

We will see below that $\left\langle\mathbf{r}\left(t_{w}\right)\right\rangle_{W}$ can take a simple form for specific choices of the system wavefunction. Nevertheless one can see qualitatively that the wavefunctions $\psi\left(t_{w}, \mathbf{r}\right)$ and $\chi\left(t_{w}, \mathbf{r}\right)$ must overlap significantly around the position of the WMA $\mathbf{r} \approx \mathbf{R}^{0}$ in order to obtain a non vanishing weak value. If $\left\langle\mathbf{r}\left(t_{w}\right)\right\rangle_{W}=0$, this essentially means there is no wavefunction exploring the region around $\mathbf{R}^{0}$ compatible with the postselected state. In the

\footnotetext{
${ }^{3}$ Since the spatial WMA wavefunction picks up an $\mathbf{R}$ dependent phase term proportional to the weak value, the WMA pointer is monitored in momentum space.
} 
special case in which $\psi\left(t_{w}, \mathbf{r}\right)$ (the preselected state propagated forward in time) and $\chi\left(t_{w}, \mathbf{r}\right)$ (the postselected state propagated backward in time) are identical, then it is easy to see that under certain conditions (eg, $\psi$ and $\chi$ are constant in the region where $f\left(\left|\hat{\mathbf{r}}-\mathbf{R}^{0}\right|^{2}\right)$ is non-zero, or have a maximum at $\left.\mathbf{R}^{0}\right)$ the weak value will be simply given by $\left\langle\mathbf{r}\left(t_{w}\right)\right\rangle_{W}=\mathbf{R}^{0}$, that is the location of the WMA.

Assume now there are several WMA of the same type distributed at positions $\mathbf{R}_{k}^{0}, k=$ $1, \ldots, N$. It is convenient to label them according to the order in which they interact $(k=1$ corresponds to the meter interacting first with the system, $k=2$ to the second meter having interacted with the system and so on). Each WMA, endowed with its own wavefunction $\phi\left(\mathbf{R}_{k}\right)$ localized around $\mathbf{R}_{k}^{0}$ interacts at time $t_{k}$ with the wavefunction through a contact interaction of the form (5) and registers a weak value

$$
\left\langle\mathbf{r}\left(t_{k}\right) ; \mathbf{R}_{k}^{0}\right\rangle_{W} \equiv \frac{\left\langle\chi\left(t_{k}\right)\left|\hat{\mathbf{r}} f\left(\left|\hat{\mathbf{r}}-\mathbf{R}_{k}^{0}\right|^{2}\right)\right| \psi\left(t_{k}\right)\right\rangle}{\left\langle\chi\left(t_{k}\right) \mid \psi\left(t_{k}\right)\right\rangle}
$$

where $\mathbf{R}_{k}^{0}$, labeling the position of the meter, will be omitted in most of the text. Overall, out of the $N$ WMA that act as meters recording the weak values, only $n$ will display a non-zero value, those for which postselection is compatible with the dynamics of the preselected state at the given WMA positions. Relabeling $k$ in a time-ordered manner reflecting the times at which the WMA have interacted, the set

$$
\mathrm{WT}_{\psi\left(t_{0}\right), \chi\left(t_{f}\right)}=\left\{t_{k}, \operatorname{Re}\left[\left\langle\mathbf{r}\left(t_{k}\right)\right\rangle_{W}\right]\right\}, \quad k=1, \ldots n
$$

defines a trajectory in the sense of weak position measurements, that is a "weak trajectory" for given pre and postselected states. Note that the pre and post-selected states $\left|\psi\left(t_{k}\right)\right\rangle$ and $\left|\chi\left(t_{k}\right)\right\rangle$ at times $t_{k}$ cannot be freely chosen but depend on the initial pre-selected state $\left|\psi\left(t_{0}\right)\right\rangle$ and on the final post-selected state $\left|\chi\left(t_{f}\right)\right\rangle$ respectively.

For an arbitrary quantum system a WT (8) will typically reflect the space-time correlation between the forward evolution of the preselected state and the backward evolution of the postselected state at the positions $\mathbf{R}_{k}^{0}$ of the weakly interacting meters: only the WMA at positions for which this correlation is non-vanishing will display a non-zero weak value. WTs become particularly interesting in the semiclassical regime, ie when the Feynman path integral is approximately given by the semiclassical propagator involving a propagator given by a coherent sum over the paths of the classical corresponding system. Indeed, as we will see below (Secs. III and IV) the weak trajectories can in principle be employed to record the 
sum over paths of the semiclassical propagator. But we will first introduce another type of trajectories that can be inferred from a different type of weak measurement.

\section{Weak measurements of momentum: velocity field}

The standard textbook form of the quantum mechanical probability current for a system in state $\psi(\mathbf{r}, t)$ is given by

$$
\mathbf{j}(\mathbf{r}, t)=\frac{i \hbar}{2 m}\left[\psi(\mathbf{r}, t) \nabla \psi^{*}(\mathbf{r}, t)-\psi^{*}(\mathbf{r}, t) \nabla \psi(\mathbf{r}, t)\right] .
$$

A local velocity field at the space-time point $(\mathbf{r}, t)$ can be defined from the current density through

$$
\mathbf{v}(\mathbf{r}, t)=\frac{\mathbf{j}(\mathbf{r}, t)}{\rho(\mathbf{r}, t)}
$$

where $\rho(\mathbf{r}, t) \equiv|\psi(\mathbf{r}, t)|^{2}$. Consider now applying the weak value definition (2) to a weak measurement of the momentum operator $\hat{\mathbf{p}}$ when the system is in state $|\psi(t)\rangle$ immediately followed by a postselection to the position eigenstate $\left|\mathbf{r}_{f}\right\rangle$. The real and imaginary parts of the weak value are given by

$$
\begin{aligned}
\langle\mathbf{p}(t)\rangle_{W} & =\frac{\left\langle\mathbf{r}_{f}|\hat{\mathbf{p}}| \psi(t)\right\rangle}{\left\langle\mathbf{r}_{f} \mid \psi(t)\right\rangle} \\
& =m \mathbf{v}\left(\mathbf{r}_{f}, t\right)-i \hbar \frac{\nabla \rho\left(\mathbf{r}_{f}, t\right)}{2 \rho\left(\mathbf{r}_{f}, t\right)}
\end{aligned}
$$

Hence by performing weak measurements of the momentum at different space-time points $\left(\mathbf{r}_{f}, t\right)$, the real part of the weak value $\langle\mathbf{p}(t)\rangle_{W}$ allows to reconstruct the velocity field $\mathbf{v}(\mathbf{r}, t)$.

Note that $\langle\mathbf{p}(t)\rangle_{W}$ can also be obtained from the difference of two position measurements made in a very small time interval [13]. One first defines a weak position measurement at time $t-\varepsilon$ consistent with postselection at position $\mathbf{r}_{f}$ at time $t$ :

$$
\langle\mathbf{r}(t-\varepsilon)\rangle_{W}=\frac{\left\langle\mathbf{r}_{f}|U(t, t-\varepsilon) \hat{\mathbf{r}}| \psi(t-\varepsilon)\right\rangle}{\left\langle\mathbf{r}_{f}|U(t, t-\varepsilon)| \psi(t-\varepsilon)\right\rangle} .
$$

To first order in $\varepsilon \rightarrow 0$ one obtains after some manipulations [13]

$$
\langle\mathbf{p}(t)\rangle_{W}=m \frac{\left(\mathbf{r}_{f}(t)-\langle\mathbf{r}(t-\varepsilon)\rangle_{W}\right)}{\varepsilon}
$$

so that the velocity field appears as the real part of the difference $\left(\mathbf{r}_{f}(t)-\langle\mathbf{r}(\mathbf{t}-\varepsilon)\rangle_{W}\right) / \varepsilon$.

It turns out [12] that this velocity field matches the local particle velocity in the de Broglie-Bohm interpretation of quantum mechanics (see Sec. III C below). The imaginary 
part of the weak value (12) has also very recently [14 been interpreted as the osmotic velocity of a putative stochastic model underlying the de Broglie-Bohm framework. The relations (11)-(12) between weak values and the Bohmian momentum are important, not only because they allow in principle to extract experimentally Bohmian trajectories from the weak measurements of the momentum velocity field, but also because these relations constitute a link between the momentum operator and the Bohmian particles momentum (the latter having little to do with the eigenvalues of the momentum operator).

\section{QUANTUM PROPERTIES AND TRAJECTORIES}

\section{A. Model system and setting}

As mentioned in the Introduction, it is often useful to interpret properties of quantum systems in terms of trajectories. This is particularly the case for quantum systems in the semiclassical regime for which the underlying classical dynamics drives the quantum evolution operator. Nevertheless a typical system in the semiclassical regime is not easy to handle - the full semiclassical propagation is generally a formidable task, the search for classical periodic orbits in general is not trivial and the computation of Bohmian trajectories calls for a powerful numerical implementation. We will work instead with a simple model system, a two dimensional time-dependent linear oscillator (TDLO). The TDLO allows to simulate the sum over paths aspect of the semiclassical propagator, given below by Eq. (26) in an

easy and tractable manner. Moreover since the TDLO Hamiltonian (15) is quadratic, the semiclassical approximation is quantum mechanically exact [1].

We shall consider in the following a two-dimensional time-dependent oscillator with timedependent frequencies $V_{x}(t)$ and $V_{y}(t)$, whose Hamiltonian is given by

$$
H(t)=\frac{\mathbf{p}^{2}}{2 m}+\frac{m}{2}\left(V_{x}(t) x^{2}+V_{y}(t) y^{2}\right)
$$

where for definiteness the time-dependence of the potential will be chosen to take the form

$$
V_{j}(t)=v_{j}-\kappa_{j} \cos \left(2 \omega_{j} t\right), \quad j=x, y
$$

(see Appendix A for details). Let us take an initial state made up of a single 2D Gaussian

$$
\psi^{\left(\mathbf{q}_{0}, \mathbf{p}_{0}\right)}\left(\mathbf{r}, t_{0}\right)=\left(\frac{2 m}{\pi \alpha_{0}^{2}}\right)^{1 / 2} e^{-m\left(\mathbf{r}-\mathbf{q}_{0}\right)^{2} / \alpha_{0}^{2}} e^{i \mathbf{p}_{0} \cdot\left(\mathbf{r}-\mathbf{q}_{0}\right) / \hbar}
$$


where $\mathbf{r}=(x, y)$, the parameters and $\mathbf{q}_{0}$ and $\mathbf{p}_{0}$ are the average values of the position and momentum operators respectively in that state; $\alpha_{0}$ sets the width of the initial Gaussian (for simplicity, the same initial width is taken along both directions). Eq. (15) is a separable problem, so the solutions to the time-dependent Schrödinger equation are readily obtained from the 1D TDLO described in Appendix A, yielding

$$
\psi^{\left(\mathbf{q}_{0}, \mathbf{p}_{0}\right)}(\mathbf{r}, t)=\psi(x, t) \psi(y, t)
$$

where each 1D wavefunction is given by Eq. A8. As discussed in Appendix A, $\psi^{\left(\mathbf{q}_{0}, \mathbf{p}_{0}\right)}(\mathbf{r}, t)$ can be written in terms of a wavefunction whose probability density has a maximum along the classical trajectory $\mathbf{q}(t)=\left(q_{x}(t), q_{y}(t)\right)$ having initial position $\mathbf{q}(t)=\mathbf{q}_{0}$ and momentum $\mathbf{p}(t)=\mathbf{p}_{0}$. The phase also depends on the momentum $\mathbf{p}(t)$ of that classical trajectory. Two other purely time-dependent functions $\alpha(t)=\left(\alpha_{x}(t), \alpha_{y}(t)\right)$ and $\phi(t)=\left(\phi_{x}(t), \phi_{y}(t)\right)$ linked to $\mathbf{q}(t)$ in the framework of Ermakov systems [cf. Eqs (A3) and (A5)] are also necessary to describe the time-dependent solution, that takes the form

$$
\begin{aligned}
\psi^{\left(\mathbf{q}_{0}, \mathbf{p}_{0}\right)}(\mathbf{r}, t) & =\left(\frac{4 m}{\pi^{2}} \operatorname{det}[\operatorname{Re}(\mathbf{M}(\alpha))]\right)^{1 / 4} \\
& e^{-[\mathbf{r}-\mathbf{q}(t)] \cdot \mathbf{M}(\alpha) \cdot[\mathbf{r}-\mathbf{q}(t)]} e^{i \mathbf{p}(t) \cdot[\mathbf{x}-\mathbf{q}(t)] / \hbar} e^{\frac{i}{2 \hbar}\left[\mathbf{p}(t) \cdot \mathbf{q}(t)-\mathbf{p}_{0} \cdot \mathbf{q}_{0}\right]} e^{-\frac{i}{2}\left[\phi(t)-\phi_{0}\right] \cdot \mathbf{r} / r} .
\end{aligned}
$$

$\mathbf{M}(\alpha)$ is a matrix defined by

$$
\mathbf{M}(\alpha)=m\left(\begin{array}{cc}
\frac{1}{\alpha_{x}(t)^{2}}-\frac{i \alpha_{x}^{\prime}(t)}{2 \hbar \alpha_{x}(t)} & 0 \\
0 & \frac{1}{\alpha_{y}(t)^{2}}-\frac{i \alpha_{y}^{\prime}(t)}{2 \hbar \alpha_{y}(t)}
\end{array}\right) .
$$

Note that $\alpha(t)$ determines the time-dependent width of the evolving wavefunction; the initial state (17) corresponds to $\alpha\left(t_{0}\right)=\left(\alpha_{0}, \alpha_{0}\right)$ and $\phi\left(t_{0}\right)=(0,0)$.

Initial states can also be superpositions of states (17); we will be find useful to consider initial states given by

$$
\psi\left(\mathbf{r}, t_{0}\right)=\sum_{J} a_{J} \psi^{\left(\mathbf{q}_{0}, \mathbf{p}_{0}^{J}\right)}\left(\mathbf{r}, t_{0}\right)
$$

that is a superposition (with normalized real weights $a_{J}$ ) of Gaussians initially localized at the same position $\mathbf{q}_{0}$ but with different initial mean momenta $\mathbf{p}_{0}^{J}$. The resulting wavefunction

$$
\psi(\mathbf{r}, t)=\sum_{J} a_{J} \psi^{\left(\mathbf{q}_{0}, \mathbf{p}_{0}^{J}\right)}(\mathbf{r}, t)
$$

is a sum of Gaussians 19 each propagating by following the guiding trajectory $\mathbf{q}^{J}(t)$. 


\section{B. Path integral and classical trajectories}

The solutions of the Schrödinger equation (A8) and (19) for the TDLO in which the wavefunction amplitude is concentrated along the trajectories of the classical corresponding system can best be seen to arise from the Feynman path integral approach. From a qualitative standpoint, the argument starts from the path integral form of the time evolution operator

$$
K\left(\mathbf{r}_{1}, \mathbf{r}_{0} ; t_{1}-t_{0}\right)=\int_{\mathbf{r}_{0}}^{\mathbf{r}_{1}} \mathcal{D} \mathbf{r}(t) \exp \frac{i}{\hbar}\left[\int_{t_{0}}^{t_{1}} \mathcal{L} d t\right]
$$

the propagator, that propagates the initial wavefunction along any conceivable path, according to

$$
\psi(\mathbf{r}, t)=\int K\left(\mathbf{r}, \mathbf{r}_{0} ; t-t_{0}\right) \psi\left(\mathbf{r}_{0}, t_{0}\right) d x .
$$

The propagator can be expressed in terms of classical trajectories when the action

$$
R\left(\mathbf{r}_{1}, \mathbf{r}_{0} ; t-t_{0}\right)=\int_{t_{0}}^{t_{1}} \mathcal{L} d t
$$

is huge relative to $\hbar$ ( $\mathcal{L}$ is the Lagrangian, given here by the $2 \mathrm{D}$ extension of Eq. A2 $)$. In that case, the integration in Eq. (23) is handled [1] with the stationary phase approximation, and the stationary points of the action are, by Hamilton's principle, the classical trajectories. $K$ then takes the generic form ${ }^{4}$

$$
K\left(\mathbf{r}_{1}, \mathbf{r}_{0} ; t_{1}-t_{0}\right)=(2 i \pi \hbar)^{-1} \sum_{k}\left|\operatorname{det} \frac{-\partial^{2} R_{k}}{\partial \mathbf{r}_{1} \partial \mathbf{r}_{0}}\right|^{1 / 2} \exp \frac{i}{\hbar}\left[R_{k}\left(\mathbf{r}_{1}, \mathbf{r}_{0} ; t_{1}-t_{0}\right)-\mu_{k}\right]
$$

where $k$ runs over all the classical trajectories connecting $\mathbf{x}_{0}$ to $\mathbf{x}_{1}$ in time $t_{1}-t_{0} . R_{k}$ is the classical action and the determinant gives the classical density of paths along the $k$ th classical trajectory, and $\mu_{k}$ are additional phases related to the number of conjugate points on the trajectory. The sole approximation made in employing the stationary phase implies neglecting the terms beyond the second order variations along the paths of least action. But for quadratic Lagrangians - such as A2 - the third order and greater order terms vanish, so that the semiclassical propagator $(26)$ is quantum-mechanically exact.

The propagator 26 accounts for the fact that the maximum of the initial wavefunction propagates along classical trajectories. The point that remains to be explained is the

\footnotetext{
4 This generic form actually assumes that the action has isolated non-degenerate stationary points, which is not the case here (a change of variables is necessary).
} 
functional form of the time-dependent wavefunction (19). Since the Hamiltonian (15) is separable, it is more straightforward to deal separately with two 1D propagators. Writing the classical action in terms of the correct dependent variables $x_{1}$ and $x_{0}$ with the help of the Ermakov phase and amplitude functions (see Appendix) leads after some tedious manipulations to

$$
\begin{aligned}
R_{c l}\left(x_{1}, x_{0} ; t_{1}-t_{0}\right) & =m\left\{\left(x_{1}^{2} \frac{\alpha^{\prime}\left(t_{1}\right)}{\alpha\left(t_{1}\right)}-x_{0}^{2} \frac{\alpha^{\prime}\left(t_{0}\right)}{\alpha\left(t_{0}\right)}\right)+\hbar \cot \left(\phi\left(t_{1}\right)-\phi\left(t_{0}\right)\right)\left(\frac{x_{1}^{2}}{\alpha^{2}\left(t_{1}\right)}+\frac{x_{0}^{2}}{\alpha^{2}\left(t_{0}\right)}\right)\right. \\
& \left.-2 x_{1} x_{0} \hbar\left[\frac{1}{\alpha\left(t_{1}\right) \alpha\left(t_{0}\right) \sin \left(\phi\left(t_{1}\right)-\phi\left(t_{0}\right)\right)}\right]\right\}
\end{aligned}
$$

(see also Ref. 25] describing a method to obtain directly the propagator from the Ermakov system solutions). The action is quadratic in $x_{0}$ so that with initial wavefunctions of the form (A9) Eq. (24) becomes a Gaussian integral quadratic in $x_{1}$; finally the classical solution is identified in the exponent with the help of Eq. (A4).

\section{De Broglie-Bohm trajectories}

According to the Bohmian (or de Broglie-Bohm) model [2, 26], a quantum system can be seen as the combination of a point-like particle guided by a pilot wave. The wavefunction plays the role of the pilot wave, and through its modulus, it also gives the statistical distribution of the particle's position, thereby recovering by construction the standard (nonrelativistic) quantum mechanical probabilities and expectation values. From a dynamical point of view, the resulting Bohmian trajectories are the streamlines of the usual probability current density flow derived from the Schrödinger equation.

If we write the wavefunction in polar form as

$$
\psi(\mathbf{r}, t)=\rho^{1 / 2}(\mathbf{r}, t) \exp (i \sigma(\mathbf{r}, t) / \hbar)
$$

the current density, Eq. (9) is given as

$$
\mathbf{j}(\mathbf{r}, t)=\frac{\rho(\mathbf{r}, t) \nabla \sigma(\mathbf{r}, t)}{m} .
$$

By replacing Eq. (29) in the Schrödinger equation it can be seen that $\rho$ and $\sigma$ obey the equation

$$
\frac{\partial \sigma}{\partial t}+\frac{(\nabla \sigma)^{2}}{2 m}+V+Q=0
$$


$V$ is the usual potential (here $\left.V=\frac{m}{2} V_{x}(t) x^{2}+\frac{m}{2} V_{y}(t) y^{2}\right)$ and the term

$$
Q(\mathbf{r}, t) \equiv-\frac{\hbar^{2}}{2 m} \frac{\nabla^{2} \rho}{\rho}
$$

is known as the quantum potential. The velocity field introduced above [Eq. [10]] gives the velocity of the Bohmian particle at the space-time point $(\mathbf{r}, t)$; it can be written in the form

$$
\mathbf{v}(\mathbf{r}, t)=\frac{\nabla \sigma(\mathbf{r}, t)}{m} .
$$

The momentum field can also be derived [27] without employing the polar form (29) as a component of the energy-momentum tensor of the Schrödinger field ${ }^{5}$.

Applying $\nabla$ to Eq. (31) and using Eq. (33) leads to

$$
m \frac{d \mathbf{v}}{d t}=-\nabla(V+Q)
$$

a Newtonian-like law of motion. This justifies, in the de Broglie-Bohm formulation, the assumption that the streamlines of the probability flow are actually trajectories taken by a point-like particle governed by Eq. (34), where the dynamics is determined not by the sole usual potential $V$ but by the a total potential function $V+Q$ thus including a wavefunctiondependent "quantum potential" term.

When the wavefunction vanishes the quantum potential becomes singular and dominates the dynamics. Therefore generically Bohmian trajectories cannot be classical [7], even in semiclassical systems. Nevertheless the signatures of the underlying classical dynamics in quantum systems, that appear as large scale structures in the quantum properties, are recovered on a statistical basis. An illustration is given immediately below. The apparent conflicting situation between the classical trajectories resulting from the path integral approach and the de Broglie-Bohm trajectories with regards to the interpretation of the dynamics of a system such as the TDLO becomes particularly acute in the context of weak measurements, as illustrated in Sec. IV]

\footnotetext{
${ }^{5}$ This is particularly appealing when generalizations are considered to relativistic quantum fields, in particular for the interpretation of experiments performed in the single photon regime as in the results reported in Ref. [16, in which the observed Bohmian-like average trajectories can be interpreted as the energy flux of the energy-momentum tensor (just like the Poynting vector appears in the stress-energy tensor for classical Maxwell fields).
} 

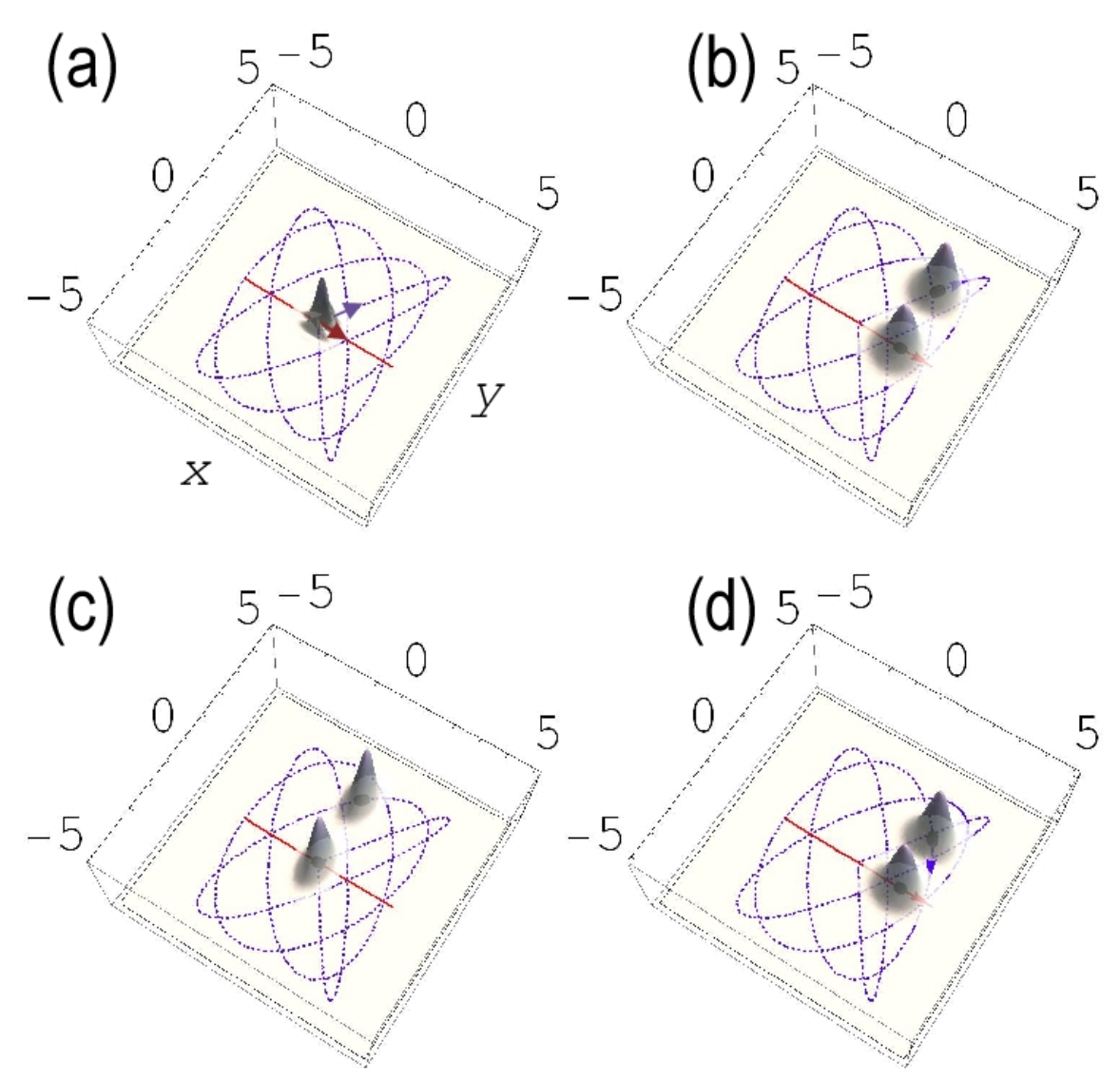

FIG. 1: (a) The probability density of the initial $\left(t_{0}=0\right)$ wavefunction Eq. 21 with $\mathbf{q}_{0}=0$, $J=1,2$ and $a_{1}=a_{2}$ is seen to be localized at the origin; the direction of $\mathbf{p}_{0}^{J}$ is schematically indicated by the arrows. The two classical trajectories with initial conditions $\left(\mathbf{q}_{0}, \mathbf{p}_{0}^{1}\right)$ and $\left(\mathbf{q}_{0}, \mathbf{p}_{0}^{2}\right)$ are shown in dashed (purple) and solid (red) lines. Both trajectories are closed at the origin. The probability density is then shown (b): at $t>t_{0}$ when the wavepackets start moving; each wavepacket follows the classical guiding trajectory $J=1,2$; (c) at $t=t_{r e c(2)}$, corresponding to the second peak of the autocorrelation (see text and Fig. 3); (d) just before the two wavepackets cross and interfere. Atomic units are used throughout, with a TDLO having unit mass.

\section{Illustration: Recurrence spectrum and returning trajectories in the TDLO}

As an illustration of the different dynamical pictures that arise depending on the nature of trajectories that are implemented, let us take our 2D time-dependent linear oscillator (TDLO). Consider an initial wavefunction given by Eq. (21) with $\mathbf{q}_{0}=0, J=1,2$ and 
$a_{1}=a_{2}{ }^{6}$; the direction of $\mathbf{p}_{0}^{J}$ is shown schematically in Fig. 1 (a) along with the resulting classical trajectories. Snapshots of the time-dependent wavepackets are shown in Fig. 1(a)(d). Each wavepacket follows the guiding trajectory $\mathbf{q}^{J}(t)$. Both of these guiding trajectories are periodic with period $4 \pi$ (the plots in Fig. 1 show the trajectories in the interval $[0,2 \pi]$ ).

Two typical Bohmian trajectories are shown in Fig. 2. The first (resp. second) Bohmian trajectory was chosen so that shortly after $t=0$, the Bohmian particle sits at the maximum of the $J=1$ (resp. $J=2$ ) wavepacket. The main characteristic of the Bohmian trajectories is that they seem to "jump" from one guiding trajectory to the other each time the wavepackets cross or interfere. This is a simple consequence of Eq. (33): the velocity of the Bohmian "particle" is proportional to the overall current density resulting from the interfering wavepackets, and by definition the current density lines do not cross each other. Although these two families of Bohmian trajectories are different from the classical guiding trajectories, on a statistical basis the motion of the wavepackets along the guiding trajectories is recovered.

The Bohmian and classical trajectories can be seen as two alternative manners of defining the propagation and transport of the probability density in this TDLO. For example if one focuses on the probability density in a small region $\mathcal{V}$ around the origin, the so-called recurrence spectrum (Fig. 3), defined by the probability $P(t)=\int_{\mathcal{V}}|\psi(\mathbf{x}, t) d \mathbf{x}|$ displays sharp peaks at specific times $t_{r e c}$. These values of $t_{r e c}$ correspond to the passage of a wavepacket in the region $\mathcal{V}$ and they are obviously given by the times at which one of the classical guiding trajectories passes through the origin. It can be seen from Figs. 3 and 1, that the first recurrence taking place at time $t_{r e c(1)}$ is due to the wavepacket propagating along the $J=2$ (red solid) guiding trajectory. Actually the first return to the origin of the $J=1$ (dashed purple) trajectory only happens at $t=t_{r e c(7)}=2 \pi$. In terms of the Bohmian trajectories, the interpretation is more involved: as can be seen from Fig. 2(b) the Bohmian trajectory plotted in green (light gray) passes through the origin at $t=t_{\text {rec (1) }}, t_{\text {rec (3) }}, t_{r e c(5)}$ and $t_{r e c(7)}=2 \pi$ and therefore contributes to the relevant peaks in the recurrence spectrum of Fig. 3. On the other hand the purple (dark gray) trajectory is not near the origin at those recurrence times, but instead contributes to the peaks at the recurrence times $t=t_{r e c(2)}, t_{r e c(4)}, t_{r e c(6)}$ and $t_{r e c(7)}$ (this is not shown in the figures). We thus see that the

\footnotetext{
${ }^{6}$ In the examples given in this work atomic units are used throughout, with a TDLO having unit mass.
} 

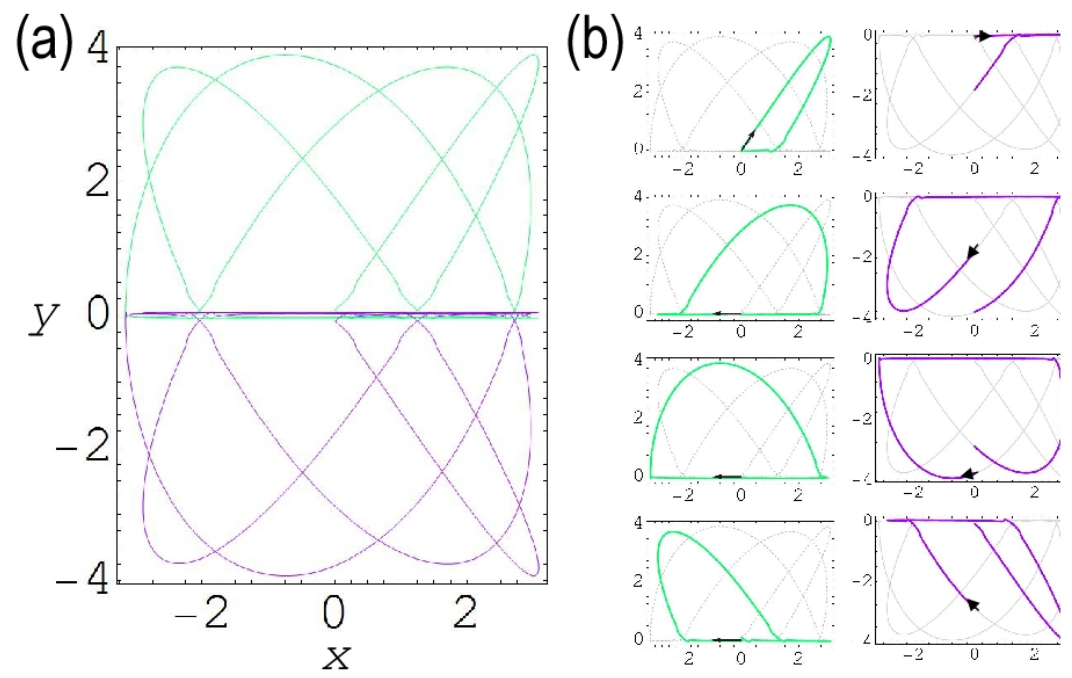

FIG. 2: (a) Two Bohmian trajectories corresponding to the wavefunction shown in Fig. 1 are plotted in light grey (green) and dark grey (purple); their initial conditions are near the maximum of the wavefunction, $\mathbf{x}_{d B B}(0)=(0.01,0.09)$ and $(0.01,-0.08)$ respectively. $(\mathrm{b})$ : The time evolution of these 2 Bohmian trajectories are depicted in the time intervals (from top to bottom): $\left(0, t_{\text {rec }(1)}\right)$, $\left(t_{r e c(1)}, t_{r e c(3)}\right),\left(t_{r e c(3)}, t_{r e c(5)}\right),\left(t_{r e c(5)}, t_{r e c(7)}\right)$, where $t_{r e c(j)}$ is the recurrence time defined by the return of a wavepacket to the origin (see text for details) and $t_{r e c(7)}=2 \pi$. The arrows indicate the direction of the Bohmian particle motion at the beginning of each time interval.

dynamical interpretation of the recurrence spectrum in terms of trajectories is quite different if couched in terms of classical trajectories or given in the de Broglie-Bohm framework.

\section{WEAK MEASUREMENTS AND TRAJECTORIES IN THE TDLO}

\section{A. General Remarks}

We give in this section derivations and specific computations for weak measurement of trajectories for the TDLO. We have seen in Sec. II that weak measurements of the position and momentum observables lead to different type of trajectories. A crucial difference in the protocols is that the "weak trajectories" are obtained from the analysis of several weak measurement apparata interacting with the system as it evolves from a given preselected state to a unique final post-selected state. Post-selection is not made after each weak measurement, but only at the end of the evolution. Instead the local average velocity 


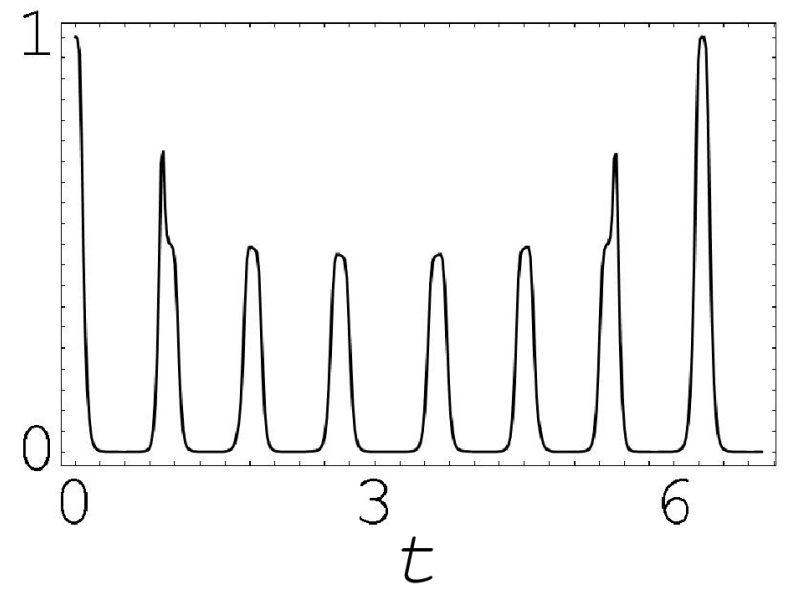

FIG. 3: The recurrence spectrum defined in the text is given as a function of time. A peak occurs when part of the wavefunction returns to the region around the origin. 7 recurrence peaks are visible in the plot, the recurrences taking place at times $t_{r e c(j)}, j=1-7$.

inferred from the weak measurement of the momentum operator is obtained by performing a single weak measurement immediately followed by post-selection; but in order to obtain the velocity field, such weak measurements must be repeated by scanning the post-selected state over the spatial region of interest.

Note also that although the pre-selected state is the initial state of the system in both cases, the post-selected states will typically be different. For "weak trajectories", it is useful to choose a post-selected state carrying the dynamical information (the mean position and momentum at the time of post-selection) of the wavepacket. The weak measurement of Bohmian trajectories relies instead on post-selecting ideally to an eigenstate of the position operator (see however Ref. [15, in which a protocol allowing to obtain an approximate weak measurement of the velocity field in non-ideal conditions is presented). 


\section{B. Weak trajectories and sum over paths}

\section{Weak trajectories and the underlying classical dynamics}

Let us start by specializing the weak position weasurements (7) to the case of the TDLO with some additional assumptions. First let us take the post-selected state to be the Gaussian

$$
\chi_{\mathbf{r}_{f}, \mathbf{p}_{f}}\left(\mathbf{r}, t_{f}\right)=\left(\frac{2}{\pi \delta_{f}^{2}}\right)^{1 / 2} e^{-\left(\mathbf{r}-\mathbf{r}_{f}\right)^{2} / \delta_{f}^{2}} e^{i \mathbf{p}_{f} \cdot\left(\mathbf{r}-\mathbf{r}_{f}\right) / \hbar}
$$

Recall from Eq. (7) that the expression of the weak position at time $t_{k},\left\langle\mathbf{r}\left(t_{k}\right)\right\rangle_{W}$ involves the post-selected state $\left|\chi\left(t_{k}\right)\right\rangle$ at time $t_{k}$ which is the wavefunction $\chi_{\mathbf{r}_{f}, \mathbf{p}_{f}}\left(\mathbf{r}, t_{f}\right)$ evolved backward in time. For the TDLO, this means finding the guiding trajectory $\mathbf{q}_{f}(t)$ having the final boundary condition $\mathbf{q}_{f}\left(t_{f}\right)=\mathbf{r}_{f}$ and $\mathbf{p}_{f}\left(t_{f}\right)=\mathbf{p}_{f}$. At time $t_{k}$ the backward evolved guiding trajectory will be found at the position $\mathbf{q}_{f}\left(t_{k}\right)$. Hence a non-vanishing weak value will be registered by a WMA positioned near $\mathbf{R}^{0} \approx \mathbf{q}_{f}\left(t_{k}\right)$ provided the wavefunction $\psi^{\left(\mathbf{q}_{0}, \mathbf{p}_{0}\right)}(\mathbf{r}, t=$ $t_{k}$ ) has a substantial amplitude in the region $\mathbf{r} \approx \mathbf{q}_{f}\left(t_{k}\right)$.

A particular case of practical interest arises when

$$
\chi_{\mathbf{r}_{f}, \mathbf{p}_{f}}\left(\mathbf{r}, t_{f}\right) \approx \psi^{\left(\mathbf{q}_{0}, \mathbf{p}_{0}^{J}\right)}\left(\mathbf{r}, t_{f}\right),
$$

where following the notation of Eqs. 21)-22 $\psi^{\left(\mathbf{q}_{0}, \mathbf{p}_{0}^{J}\right)}\left(\mathbf{r}, t_{f}\right)$ represents the branch of the pre-selected wavefunction $\psi\left(\mathbf{r}, t_{0}\right)=\sum_{J} a_{J} \psi^{\left(\mathbf{q}_{0}, \mathbf{p}_{0}^{J}\right)}\left(\mathbf{r}, t_{0}\right)[$ Eq. 21)] propagating along a guiding trajectory $\mathbf{q}^{J}(t)$. Then only a WMA positioned along the guiding trajectory $\mathbf{q}^{J}(t)$ will display non-vanishing weak values. Moreover since Eq. (36) then holds for any $t$, each weak value $\left\langle\mathbf{r}\left(t_{k}\right)\right\rangle_{W}$ becomes an expectation value of the position in the state $\psi^{\left(\mathbf{q}_{0}, \mathbf{p}_{0}^{J}\right)}\left(\mathbf{r}, t_{k}\right)$, which is simply the real term ${ }^{7}$

$$
\left\langle\mathbf{r}\left(t_{k}\right)\right\rangle_{W}=\mathbf{q}^{J}\left(t_{k}\right)
$$

The corresponding weak trajectory (8) is hence the guiding trajectory $\mathbf{q}^{J}(t)$

$$
\mathrm{WT}_{\psi\left(t_{0}\right), \chi\left(t_{f}\right)}=\left\{t_{k}, \operatorname{Re}\left\langle\mathbf{r}\left(t_{k}\right)\right\rangle_{W}\right\}=\left\{t_{k}, \mathbf{q}^{J}\left(t_{k}\right)\right\}
$$

Note that in order to obtain (38), Eq. (36) is a sufficient but non-necessary condition. In particular, it can be deduced from Eq. 40 given below that any $\chi_{\mathbf{r}_{f}, \mathbf{p}_{f}}\left(\mathbf{r}, t_{f}\right)$ such that $\mathbf{q}^{J}\left(t_{f}\right)=\mathbf{q}_{f}\left(t_{f}\right)$ and $\mathbf{p}^{J}\left(t_{f}\right)=\mathbf{p}_{f}\left(t_{f}\right)$ will also lead to the weak trajectory 38 .

\footnotetext{
${ }^{7}$ This is not valid for the WMAs located at a point where guiding trajectories cross.
} 

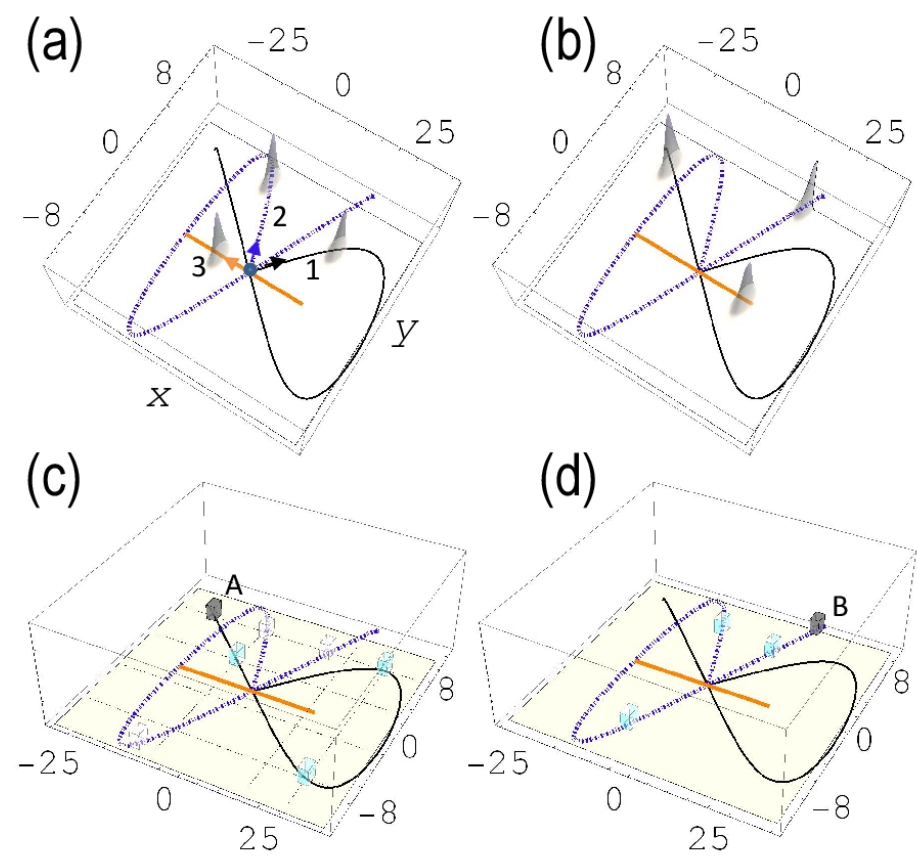

(d)

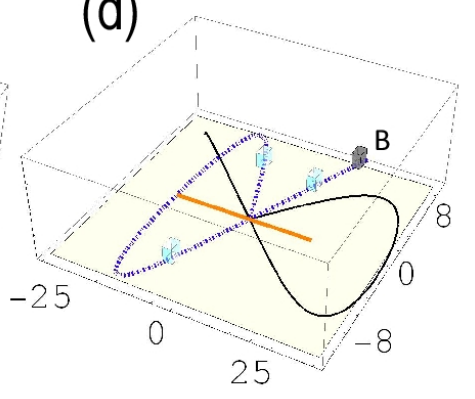

FIG. 4: (a) A wavefunction of the form given by Eq. (21) with $\mathbf{q}_{0}=0, J=1,2,3, a_{1} \approx a_{2} \approx a_{3}$, and with $\mathbf{p}_{0}^{J}$ schematically indicated by the arrows, initially $\left(t_{0}=0\right)$ compactly localized at the origin (blue dot) propagates along the the 3 guiding trajectories $J$ (see labels in the plot). The probability density is shown at $t>t_{0}$ when the wavepackets start moving along the guiding trajectories. (b) The probability density is shown at $t=t_{f}$, the time at which the postselection is made. (c) Postselection, represented by the gray box, is made at point $A$ along the $J=1$ trajectory with a postselection to state $(38)$ with $J=1$. A grid of WMAs (weak meaurement apparata, acting as quantum probes) as defined in the text is suggested, some WMAs being explicitly pictured (white boxes). Only the WMAs along trajectory $A$ have their quantum state modified (indicated by a blue shading) whereas the quantum states of the other WMAs on the grid are left unchanged. The weak trajectory can be inferred by reading the states of the WMAs. (d) Same as (c) when postselection is made at point $B$ along the $J=2$ trajectory with a postselected state (38) with $J=2$ : only the WMAs along trajectory $B$ (shown by a blue shading) have their quantum state modified, with a phase term proportional to the weak value (37).

An illustration is given in Fig. 4. An initial wavefunction

$$
\psi\left(\mathbf{r}, t_{0}\right)=\sum_{J=1}^{3} a_{J} \psi^{\left(\mathbf{q}_{0}=0, \mathbf{p}_{0}^{J}\right)}\left(\mathbf{r}, t_{0}\right)
$$

tightly localized at the origin, subsequently expands as a sum over paths involving 3 guiding 
trajectories $J=1,2,3$ [Fig. 4(a)]. Post-selection will take place at $t=t_{f}$ chosen slightly after the wavepackets have returned for the first time to the origin [the probability density at $t=t_{f}$ is plotted in 4(b)]. Let us first choose a post-selected state given by Eq. (36) with $\mathbf{r}_{f}=\mathbf{r}_{A}$ [the point $A$ is displayed in Fig $4(\mathrm{c})$ ] and $J=1$. Assume a set of WMA was disposed on a grid as indicated schematically in Fig 4(c). Then, only the WMAs placed along the classical trajectory $\mathbf{q}^{J=1}(t)$ record a non-vanishing weak value. This weak value is given by Eq. (37). Fig 4(d) shows the WMA having a non-vanishing weak-value for the same preselected state but for a postselected state obeying (36) at $\mathbf{r}_{f}=\mathbf{r}_{B}$ with $J=2$ : those WMAs are precisely the ones placed along the classical trajectory $\mathbf{q}^{J=2}(t)$.

\section{Isolated weak values}

$\left\langle\mathbf{r}\left(t_{k}\right)\right\rangle_{W}$ can also be obtained analytically for a post-selected state of the form 35 but not obeying the condition $(36)$. Provided a WMA does not lie at positions where different branches $\psi^{\left(\mathbf{q}_{0}=0, \mathbf{p}_{0}^{J}\right)}(\mathbf{r}, t)$ of the system wavefunction overlap, and further assuming that the wavepackets are narrower than the range of the system-WMA interaction given by

$f\left(\left|\hat{\mathbf{r}}-\mathbf{R}_{k}^{0}\right|^{2}\right)$, the integrals in Eq $\sqrt[77]{ }$ defining the weak value can be computed analytically and where non-vanishing put in the compact form

$$
\left\langle\mathbf{r}\left(t_{k}\right)\right\rangle_{W}=\mathbf{q}^{J_{k}}\left(t_{k}\right)+\left[\mathbf{q}^{J_{k}}\left(t_{k}\right)-\mathbf{q}_{f}\left(t_{k}\right)\right] \cdot \mathbf{m}_{1}\left(t_{k}\right)+\left[\mathbf{p}^{J_{k}}\left(t_{k}\right)-\mathbf{p}_{f}\left(t_{k}\right)\right] \cdot \mathbf{m}_{2}\left(t_{k}\right) .
$$

As above $\mathbf{q}^{J_{k}}\left(t_{k}\right)$ and $\mathbf{p}^{J_{k}}\left(t_{k}\right)$ are the position and momentum of the classical guiding trajectory $J_{k}$ at the time the system interacts with the $k$ th WMA. $\mathbf{q}_{f}\left(t_{k}\right)$ and $\mathbf{p}_{f}\left(t_{k}\right)$ are the position and momentum at time $t_{k}$ of the trajectory having the boundary conditions fixed by the choice of post-selection, $\mathbf{q}_{f}\left(t_{f}\right)=\mathbf{r}_{f}$ and $\mathbf{p}_{f}\left(t_{f}\right)=\mathbf{p}_{f}$ (so that $\mathbf{q}_{f}(t)$ is formally the trajectory with the final boundary conditions determined by postselection evolved backward in time). $\mathbf{m}_{1}\left(t_{k}\right)$ and $\mathbf{m}_{2}\left(t_{k}\right)$ are purely time-dependent complex functions.

Note that there is now an index $k$ at $J_{k}$ given that Eq. (36) is not obeyed. In that case if postselection at $t_{f}$ happens on, a branch, say $J_{f}$ of the wavefunction, a nonzero weak value $\left\langle\mathbf{r}\left(t_{k}\right)\right\rangle_{W}$ can only be obtained provided the backward evolving $\mathbf{q}_{f}(t)$ crosses "accidentally", at some time $t_{k}$ a wavepacket moving along another branch, denoted $J_{k}$ (thereby evolving along the classical trajectory $\mathbf{q}^{J_{k}}(t)$ ). At some other time $t_{k^{\prime}}$, one can imagine that a different trajectory $\mathbf{q}^{J_{k^{\prime}}}(t)$ may be crossed, especially if the inital wavefunction of the form (39) 
contains many branches. In this situation, the weak values will be nonzero for a small number of isolated points. Compared to the previous case, where a weak trajectory can be inferred from (38) involving in principle a dense number of closely positioned WMAs, the set $\left\{t_{k}, \mathbf{R}_{k}^{0}\right\}$ containing a few points cannot be said to form a trajectory, but rather isolated points belonging to different branches of the wavefunction. An exemple is given in Fig. 5(a).

When the conditions stated above Eq. (40) are not fulfilled, then $\left\langle\mathbf{r}\left(t_{k}\right)\right\rangle_{W}$ must be computed numerically, though the compatibility condition for obtaining non-zero weak values (overlap of $\chi_{\mathbf{r}_{f}, \mathbf{p}_{f}}\left(\mathbf{r}, t_{k}\right)$ and $\psi\left(\mathbf{r}, t_{0}\right)$ in the neighborhood of $\left.\mathbf{R}_{k}^{0}\right)$ can generally be inferred from the classical dynamics that determine the guiding trajectories.

\section{Sum over paths}

We have just seen that when post-selection takes place along a given branch of the wavefunction, then if the postselected state is dynamically compatible with the guiding classical trajectory carrying that branch, then only the WMAs placed along that guiding trajectory will display non-zero weak values. Post-selecting appropriately on a different branch will instead yield non-zero weak values along the guiding trajectory associated with that specific branch. Now since the wavefunction (39), or more generically the semiclassical propagator (26) involves a sum over paths, it would be valuable if the corresponding weak trajectories could be detected simultaneously by the weak measurement apparata.

This is possible if post-selection is made at some final position $\mathbf{r}_{f}$ where two or more trajectories cross and provided the post-selected state can be tailored to take the approximate form

$$
\chi_{\mathbf{r}_{f}}\left(\mathbf{r}, t_{f}\right) \approx \sum_{K} c_{K} \chi_{\mathbf{r}_{f}, \mathbf{p}_{f}^{K}}\left(\mathbf{r}, t_{f}\right)
$$

where the $c_{K}$ are arbitrary coefficients and the $\chi_{\mathbf{r}_{f}, \mathbf{p}_{f}^{K}}\left(\mathbf{r}, t_{f}\right) \approx \psi^{\left(\mathbf{q}_{0}, \mathbf{p}_{0}^{K}\right)}\left(\mathbf{r}, t_{f}\right)$ as in Eq. (36). Plugging in Eqs. (22) and (41) in the weak value definitions (1) and (7) yields

$$
\begin{aligned}
\left\langle\mathbf{r}\left(t_{k} ; \mathbf{R}_{k}^{0}\right)\right\rangle_{W} & =\frac{\left\langle\chi_{\mathbf{r}_{f}}\left(t_{f}\right)\right| U\left(t_{f}, t_{k}\right) \hat{\mathbf{r}} f\left(\left|\hat{\mathbf{r}}-\mathbf{R}_{k}^{0}\right|^{2}\right)\left[U\left(t_{k}, t_{0}\right)\left|\psi\left(t_{0}\right)\right\rangle\right]}{\left\langle\chi_{\mathbf{r}_{f}}\left(t_{f}\right)\left|U\left(t_{f}, t_{0}\right)\right| \psi\left(t_{0}\right)\right\rangle} \\
& =\frac{\sum_{K, J} c_{K}^{*} a_{J}\left\langle\chi_{\mathbf{p}_{f}^{K}}\left(t_{k}\right)\left|\hat{\mathbf{r}} f\left(\left|\hat{\mathbf{r}}-\mathbf{R}_{k}^{0}\right|^{2}\right)\right| \psi^{\left(\mathbf{q}_{0}, \mathbf{p}_{0}^{J}\right)}\left(t_{k}\right)\right\rangle}{\sum_{K, J} c_{K}^{*} a_{J}\left\langle\chi_{\mathbf{p}_{f}^{K}}\left(t_{k}\right) \mid \psi^{\left(\mathbf{q}_{0}, \mathbf{p}_{0}^{J}\right)}\left(t_{k}\right)\right\rangle} .
\end{aligned}
$$




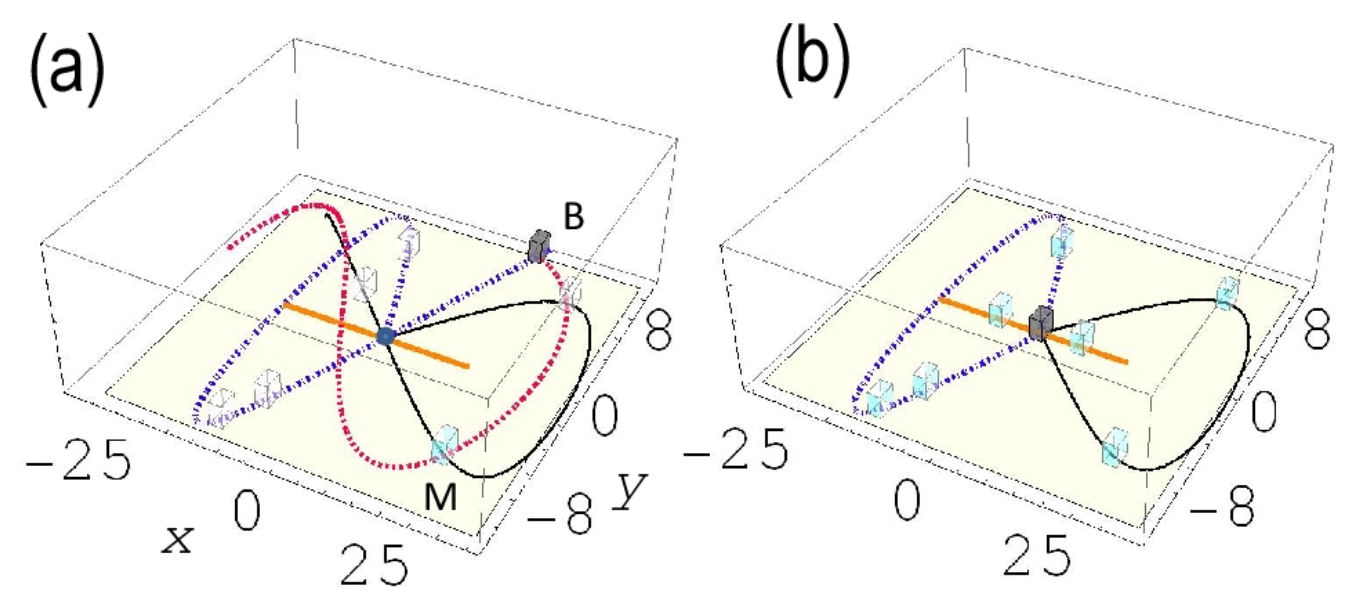

FIG. 5: (a) Isolated weak value : Postselection takes place at point $B$ along the $J=2$ trajectory, but unlike the case pictured in Fig. 4(d), the postselected state is of the form 35 but does not obey the condition (36); instead the postselected momentum is "arbitrary", and corresponds to the trajectory evolving backward in time shown in red (dashed grey). Then the WMAs placed in a grid are generally not modified (a few are shown in the figure, left unshaded) since the weak values vanish and no weak trajectory can be defined. If the post-selected backward-evolved guiding trajectory crosses accidentally a wavepacket traveling on one of the system's guiding trajectories, the weak value will be a complex number and the relevant WMA will be modified, as indicated by the light blue shading of the WMA labeled $M$. This gives rise to an isolated WMA being modified, and hence no weak trajectory can be defined. (b) Sum over paths: Post-selection takes place at $\mathbf{r}_{f}=0$ when the wavefunction first returns to the origin (grey box), with a post-selected state of the form (41). The WMAs along the three guiding trajectories $J=1,2,3$ are modified (shaded boxes) each indicating a weak value $\mathbf{q}^{J}\left(t_{k}\right)$ given by (44) (the state of the other WMAs on the grid - not shown on the figure - is left unchanged). This allows to infer weak trajectories corresponding to the sum over paths appearing in the Feynman propagator.

Assuming as we have done up to now that the WMAs set at places where the different branches interfere are disregarded, a typical WMA positioned at $\mathbf{R}_{k}^{0}$ will therefore interact at most with the wavepacket propagating along a given branch, say $J$. In turn only the same branch $J$ of the postselected state $(41)$ will overlap with the system wavefunction at $\mathbf{R}_{k}^{0}$ and 
Eq. (43) becomes

$$
\left\langle\mathbf{r}\left(t_{k}\right) ; \mathbf{R}_{k}^{0} ; J\right\rangle_{W}=\frac{\left\langle\chi_{\mathbf{p}_{f}^{J}}\left(t_{k}\right)\left|\hat{\mathbf{r}} f\left(\left|\hat{\mathbf{r}}-\mathbf{R}_{k}^{0}\right|^{2}\right)\right| \psi^{\left(\mathbf{q}_{0}, \mathbf{p}_{0}^{J}\right)}\left(t_{k}\right)\right\rangle}{\left\langle\chi_{\mathbf{p}_{f}^{J}}\left(t_{k}\right) \mid \psi^{\left(\mathbf{q}_{0}, \mathbf{p}_{0}^{J}\right)}\left(t_{k}\right)\right\rangle}=\mathbf{q}^{J}\left(t_{k}\right) ;
$$

it is now necessary to explictly state the branch $J$ relevant to the weak value. Indeed, possibly at the same time a different WMA positioned at $\mathbf{R}_{k^{\prime}}^{0}$ will have interacted with another branch $J^{\prime}$ of the system wavefunction consistent with the postselection condition (41), recording the weak value $\left\langle\mathbf{r}\left(t_{k}\right) ; \mathbf{R}_{k^{\prime}}^{0} ; J^{\prime}\right\rangle_{W}$. If there is a sufficient number of WMAs it is then straightforward to arrange the non-zero weak values extracted from the weak measurement apparata into time-ordered sets corresponding to different trajectories

$$
\begin{aligned}
& \mathrm{WT}_{\psi\left(t_{0}\right), \chi\left(t_{f}\right)}\left(J_{1}\right)=\left\{t_{k}, \operatorname{Re}\left\langle\mathbf{r}\left(t_{k}\right) ; \mathbf{R}_{k}^{0} ; J_{1}\right\rangle_{W}\right\}=\left\{t_{k}, \mathbf{q}^{J_{1}}\left(t_{k}\right)\right\} \\
& \mathrm{WT}_{\psi\left(t_{0}\right), \chi\left(t_{f}\right)}\left(J_{2}\right)=\left\{t_{k}, \operatorname{Re}\left\langle\mathbf{r}\left(t_{k}\right) ; \mathbf{R}_{k}^{0} ; J_{2}\right\rangle_{W}\right\}=\left\{t_{k}, \mathbf{q}^{J_{2}}\left(t_{k}\right)\right\} \ldots
\end{aligned}
$$

These trajectories resulting from the weak measurements of the position are a subset of the sum over paths constitutive of the propagating wavefunction compatible with the postselected state 41.

Consider for example the situation previously shown in Fig. 4 and assume postselection is made at $t_{f}=t_{r}$, when the wavepackets return for the first time to the origin $\mathbf{r}_{f}=0$. The situation is represented in Fig. 5(b): assuming a set of weakly interacting measurement apparata (WMA) laid out in a grid, the WMAs placed along the 3 guiding trajectories $J=1,2$ and 3 will have their quantum state modified according to the weak value $\mathbf{q}^{J}\left(t_{k}\right)$. By retrieving the weak values, a set of weak trajectories corresponding to the three classical guiding trajectories along which the wavepackets move can be defined. This shows that by postselecting appropriately at a position where several Feynman paths cross, it is in principle possible to observe the sum over paths as weak trajectories resulting from the interaction between WMAs and the system wavefunction.

\section{Weak measurement of the velocity field and Bohmian trajectories}

As we have seen above [Eqs. (11)-(12)], the weak measurement of the momentum operator followed immediately by postselection to an eigenstate $\left|\mathbf{r}_{f}\right\rangle$ of the position operator yields a velocity field that turns out to correspond to the local velocity at $\mathbf{r}_{f}$ of the particle 


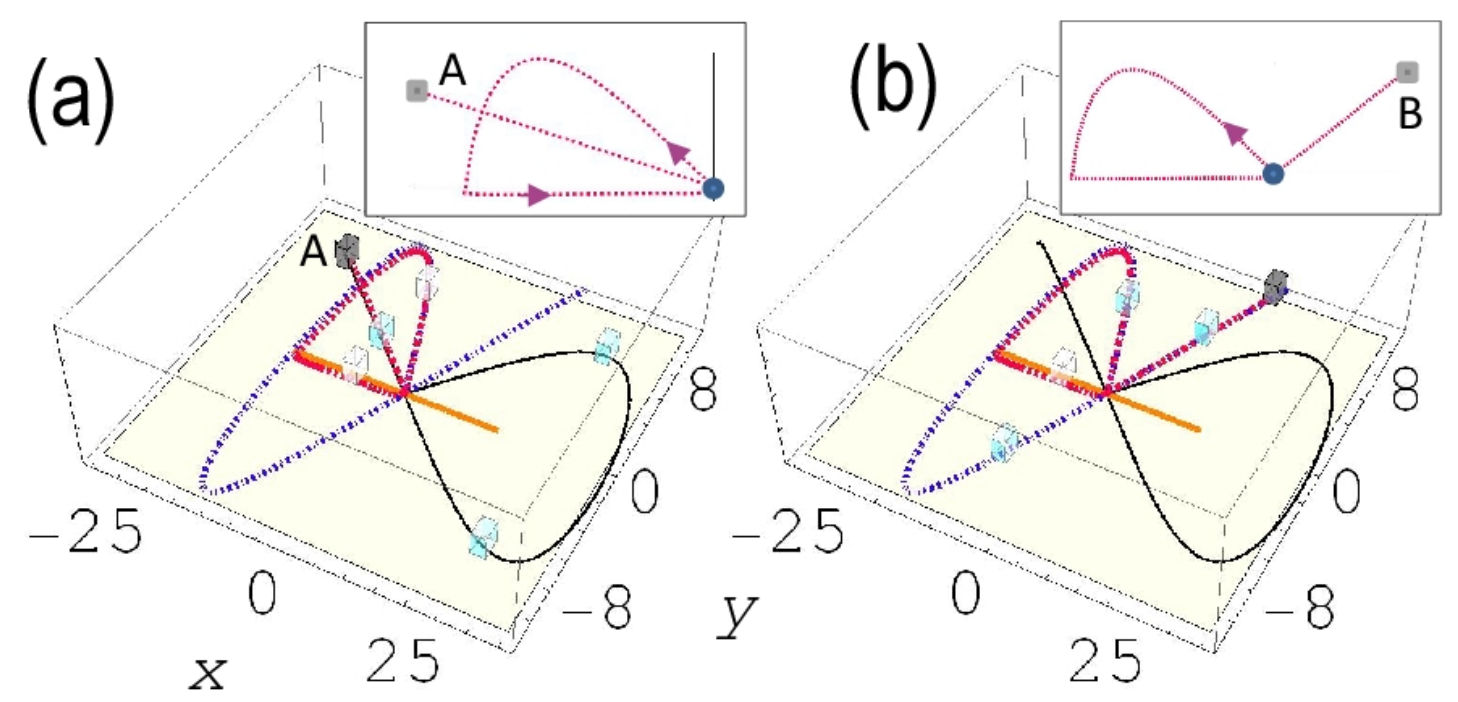

FIG. 6: (a) A typical Bohmian trajectory with its final $\left(t=t_{f}\right)$ position at the postselection point $A$ (on the $J=1$ guiding trajectory) is shown (dark grey [online:red] thick dashed line). The postselection condition is the one illustrated in Fig 4(c), for which the WMAs that have interacted (shaded in the figure) are only along the $J=1$ trajectory (all the other WMAs, including those along the Bohmian trajectory, are left unshaded). The inset details the Bohmian trajectory, starting at the origin, first following the wavepacket travelling along the $J=2$ guiding trajectory then jumping to the wavepacket along the $J=3$ guiding trajectory, going back to the origin and leaving along the wavepacket following the $J=1$ trajectory. All the Bohmian trajectories with a final position in the vicinity of $A$ have the form shown in the inset. (b): Same as (a) but for a postselection condition at $t=t_{f}$ at point $B$, corresponding to the example shown in Fig 4 (d). A typical Bohmian trajectory having its endpoint in the vicinity of $B$ is shown [dark grey (online:red) thick dashed line]. Here the shaded WMAs are those along the $J=2$ guiding trajecotry. The inset details how the Bohmian trajectory unfolds in time.

constitutive of the Bohmian model characterized by the law of motion (34). Here each weak measurement, made by a single WMA positioned at $\mathbf{R}^{0}=\mathbf{r}_{f}$, is followed by a projective measurement ideally at the same position. The projective measurement terminates the system evolution, so the procedure must be repeated first for the same $t$ throughout space (or at least where the wavefunction amplitude is known to be non-negligible) and this must be done again for each value of $t$ under consideration. Overall, these weak measurements allow to map unambiguously the velocity field $\mathbf{v}\left(\mathbf{r}_{f}, t\right)=\operatorname{Re}\langle\mathbf{p}(t)\rangle_{W} / m$ at each space-time point. 
Note that strictly speaking, it is not possible to deduce unambiguously particle trajectories from a finite sample of velocity field values $\mathbf{v}\left(\mathbf{r}_{f}, t_{k}\right)$, without assuming in the first place that the streamlines correspond to the actual motion of particles. The additional specific assumption (34) needs to be made. Then the Bohmian trajectories can be integrated from the velocity field. The Bohmian trajectories shown in Fig. 6 have been computed by numerical integration. They correspond to the wavefunction displayed in Fig. 4.

Fig. 6(a) shows a typical Bohmian trajectory consistent with postselection at point $A$ [see Fig 4(c)]. In order to infer that Bohmian trajectory from experimental observations, one would need to repeat the weak measurement procedure of Sec. II C by taking different postselection points at different times. Instead in Fig. 6(a) we directly show a Bohmian trajectory arriving at point $A$ in the the postselection region. If we assume we have carried out the postselection at $A$ as specified in Fig. 4(c), then only the WMAs along trajectory $J=1$ will be shaded, the other WMAs on the grid do not have their quantum state modified. The trajectory shown in Fig. 6(a) is the one that at $t=t_{f}$ has the final position $\mathbf{x}_{d B B}\left(t_{f}\right)=\mathbf{q}^{J=1}\left(t_{f}\right)$ ie at the maximum of the wavepacket that has followed the guiding trajectory $J=1$. This trajectory is "typical" in the sense that all the other Bohmian trajectories having the position at $t=t_{f}$ in the vicinity of the postselected point $\mathbf{q}^{J=1}\left(t_{f}\right)$ have the same topology, leaving initially the origin along with the wavepacket travelling along $J=2$, then jumping to the wavepacket along $J=3$, going back to the origin then leaving the origin along the wavepacket following the $J=1$ trajectory [see the inset in Fig. 6(a)]. The important feature is that these Bohmian trajectories are unrelated to the weak trajectory measurements: for example the WMAs on path $J=1$ in the $x>0$ plane have interacted with the system (since their quantum state has been modified), although the Bohmian trajectories detected at $A$ do not reach these WMAs. Conversely as seen in Fig. 6(a) there are WMAs that are left unchanged (that are interpreted as not having interacted with the system given the postselection, represented by the unshaded boxes) on the path of the Bohmian trajectories.

Fig. 6(b) illustrates the same type of situation shown in Fig. 6(a) but for a weak trajectory postselected at point $B$ under the conditions depicted in Fig 4 (d). The typical Bohmian trajectory displayed in the figure ending at $B$ at the postselection time $t=t_{f}$, is not related to the shaded and unshaded WMAs, having interacted (or not) with the system along the weak trajectories. 


\section{DISCUSSION AND CONCLUSION}

The starting point of this paper was to note that when trajectories are employed to interpret the dynamics of quantum systems, the classical trajectories of the semiclassical path integral propagator on the one hand and the de Broglie Bohm trajectories on the other give rise to different accounts of the dynamics taking place in the system. While this is not a problem if these types of trajectories are envisaged as computational tools or mathematical artifacts, the main idea developed in this work was to introduce weak measurements in order to implement a practically non-disturbing observational windows that would allow to follow the evolution of the system. This was done by employing a model, a 2D time-dependent linear oscillator, introduced as a manner of simulating a more complex (but less tractable) system in the semiclassical regime.

We have seen that the classical Feynman trajectories can be observed by weakly measuring the position of the system by an array of weakly interacting devices, followed by a single postselection. Taking an arbitrary postselection state does not yield weak trajectories, given that then none of the WMAs will show signs of interaction with the system (their quantum state has not been modified, except accidentally, giving rise to an isolated weak value). The dynamical compatibility condition requires that the backward postselected state overlaps with a trajectory of the propagator. Only then will the entire set of WMAs along that trajectory indicate they have interacted with the system (their quantum state being modified by the corresponding weak value). In this sense, the postselected state must contain the information on the Feynman path appearing as a weak trajectory. Alternatively, a trial and error procedure sampling the parameter space for the postselection state can be employed, monitoring the states of the WMAs until they indicate a continuous trajectory. This will precisely be the weak trajectory of a classical Feynman propagator path.

We also saw that Bohmian trajectories can be inferred from the weak measurement of momentum. This is particularly noteworthy, given that the Bohmian momentum is not directly connected to the eigenvalues of the momentum operator. Several weak measurements must be done by sampling all the spatial domain, and this must be repeated at each time in order to follow the evolution of the flow. Defining Bohmian trajectories from the velocity requires an additional assumption, associating the motion of particle with the lines of the current density. In this sense, the weak measurements do not directly yield the observation 
of the Bohmian trajectories, but that of the momentum field.

As pointed out above [Eq. (14)], the weak measurement of momentum usually relies on inferring a velocity from the difference between two position measurements. In practice it is not possible to do the position measurements with infinite precision, and it has recently [15] been suggested to model the finite resolution using the POVM (Positive Operator valued Measure) framework. The authors of Ref. [15] have in particular shown under which conditions position Gaussian measurement operators allow to recover the Bohmian field velocity. In the context of weak trajectories, post-selection to an ideally well-known position eigenstate is not required, but Gaussian measurement operators could be useful to model post-selection to a state having the characteristics of the final wavepacket $\left|\chi\left(t_{f}\right)\right\rangle$.

So can weak measurements open an observational window that would allow to give a "correct" account of the dynamics of a quantum system in terms of trajectories? There are certainly different answers that can be given to this question, ultimately relying on interpretational commitments (including the meaning of the weak values). A consensual option (having a Copenhagen interpretation flavour) would state that the type of trajectories that one sees depends on the context - the type of weak measurements that are being made and hence the entire experimental setup including the WMAs (and in particular in their interaction with the system); such an answer would obviously undermine the idea that there is a meaningful underlying dynamics that can be understood in terms of trajectories, as these would be relegated to being artifacts or computational tools.

Still, the fact that weak trajectories are obtained from a series of weak measurements of positions given a final postselected state, as opposed to being inferred from a mean velocity field at each point, is a natural feature with regard to the definition of a space-time trajectory. Indeed, if we take the weak values in the original [8] sense as referring to a generalized value for an observable obtained without appreciably disturbing the system evolution, the weak values of the position for a chosen postselection suffice in order to extract a trajectory. On the other hand the observation of the momentum weak value at a postselected spacetime point does not as such allow to observe a trajectory, but the local weak value of the momentum field.

Moreover, sticking to the situation illustrated in Fig. 6(a), it is noticeable that there is no correlation between the Bohmian trajectories detected at the postselection point $A$ and the WMAs that interacted with the system (along parts of the $J=1$ guiding trajectory in 
which there are no Bohmian trajectories reaching $A$ ) and those that haven't (the Bohmian trajectories do not trigger the WMAs along part of their route although the interaction involves the position). This fact could apparently be taken as an argument against the relevance of interpreting the dynamics in terms of Bohmian trajectories, on the ground that the particle does not comply with its role (which is to make detectors click). This is not the case, however: one should indeed bear in mind that the weak interactions are unitary and do not translate as clicks until the WMAs themselves are measured. In addition, given the nonlocal character of the de Broglie Bohm dynamics, the internal states of the WMAs must be taken into account explicitly, and this may affect non-locally some features of the Bohmian dynamics, in particular the "no-crossing" rule [28]. While it is well-known that Bohmian trajectories are modified in genuine open systems relative to the Bohmian trajectories of the closed system (the ones we were interested in throughout this work), the extent to which this aspect subsists in the case of weak measurements remains to be investigated.

\section{Appendix A: Closed form solutions for the time-dependent harmonic oscillator}

The Hamiltonian for a one dimensional linear oscillator of mass $m$ with a time-dependent frequency $V(t)$ is given by

$$
H(t)=\frac{p^{2}}{2 m}+\frac{m}{2} V(t) q^{2}
$$

We will not distinguish in the notation the classical Hamiltonian and phase-space variables from the corresponding quantum Hamiltonian and operators unless required by the context. The solutions of the Schrödinger equation can be obtained exactly by employing different methods, like algebraic methods [29], path integrals [25] or the more popular procedure based on solving for the eigenfunctions of dynamical invariants [30]. We will employ a version [31]

of the latter method involving solutions of Ermakov systems, which is known to be well suited to working with Gaussian wavefunctions.

The Lagrangian corresponding to the Hamiltonian (A1) is

$$
\mathcal{L}=\frac{m}{2} \dot{q}^{2}-\frac{m}{2} V(t) q^{2}
$$

and leads to the classical equation of motion

$$
\partial_{t}^{2} q(t)+V(t) q(t)=0
$$


Employing an amplitude-phase decomposition of the classical solution $q(t)$ in the form

$$
q(t)=\alpha(t)\left(c_{1} \cos \left(\phi(t)-\phi\left(t_{0}\right)\right)+c_{2} \sin \left(\phi(t)-\phi\left(t_{0}\right)\right)\right)
$$

where $\alpha(t)$ is the amplitude and $\phi(t)$ the phase leads to an auxiliary nonlinear equation

$$
\frac{\partial_{t}^{2} \alpha(t)}{\alpha(t)}+V(t)=\frac{c_{0}^{2}}{\alpha^{4}(t)}
$$

along with the condition

$$
\partial_{t} \phi(t)=\frac{c_{0}}{\alpha^{2}(t)} .
$$

Eqs. (A3)-A5) form an Ermakov system [32] linking the solutions of a linear and a nonlinear equation. The $c_{i}$ in the equations above denote real constants.

The reason for introducing Ermakov systems is that the time-dependent Schrödinger equation with the Hamiltonian A1,

$$
i \hbar \partial_{t} \psi(x, t)=\left[-\frac{\hbar^{2}}{2 m} \partial_{x}^{2}+\frac{m}{2} V(t) x^{2}\right] \psi(x, t)
$$

admits the closed form solution

$$
\begin{aligned}
\psi^{\left(q_{0}, p_{0}\right)}(x, t) & =\left(\frac{2 m}{\pi \alpha^{2}(t)}\right)^{1 / 4} e^{-[x-q(t)]^{2}\left(\frac{m}{\alpha(t)^{2}}-\frac{i m \alpha^{\prime}(t)}{2 \hbar \alpha(t)}\right)} \\
& e^{i p(t)[x-q(t)] / \hbar} e^{\frac{i}{2 \hbar}\left[p(t) q(t)-p_{0} q_{0}\right]} e^{-\frac{i}{2}\left[\phi(t)-\phi_{0}\right]} .
\end{aligned}
$$

Hence the exact solutions to the Schrödinger equation can be obtained from the knowledge of the solutions to the Ermakov system, namely $q(t)$ which is the solution of the linear equation (that is also the classical equation of motion) and $\alpha(t)$ (and its integral $\phi(t)$ ) which is a solution of the nonlinear Ermakov equation. It can be checked explicitly by plugging in Eq. A8 into Eq. A7 requires setting the constant $c_{0}$ of Eq A5 to $c_{0}=2 \hbar$. Finally, the notation $q_{0}$ etc. indicates the values of the functions at $t=t_{0}$, ie $q_{0} \equiv q\left(t_{0}\right)$ and so on. Let us choose specifically the amplitude function such that $\alpha^{\prime}\left(t_{0}\right)=0$. Then at $t=t_{0}$ the initial wavefunction $\mathrm{A} 8 \mathrm{~B}$ is given by

$$
\psi^{\left(q_{0}, p_{0}\right)}\left(x, t_{0}\right)=\left(\frac{2 m}{\pi \alpha_{0}^{2}}\right)^{1 / 4} e^{-m\left[x-q_{0}\right]^{2} / \alpha_{0}^{2}} e^{i p_{0}\left[x_{0}-q_{0}\right] / \hbar},
$$

that is a standard Gaussian. $q_{0}$ and $p_{0}$ that appeared as parameters in Eq. A8 thus correspond to the average initial position and momentum of the initial Gaussian and $\alpha_{0}$ sets 
the initial width. Note that the initial choice of $q_{0}$ and $p_{0}$ also sets the values of $c_{1}$ and $c_{2}$ in Eq. A4, equal respectively to $q_{0} / \alpha_{0}$ and $p_{0} \alpha_{0} / 2 \hbar m$.

From Eq. (A8) it appears that the exact solution of the Schrödinger equation with the initial condition A9 has at all times its maximal probability along the curve $q(t)$ : this defines the guiding trajectory. The evolution of the wavefunction $\psi^{\left(q_{0}, p_{0}\right)}(x, t)$ depends on the properties of the guiding trajectory (which as we have seen above, turns out to be the solution obtained by solving the classical equations with the Hamiltonian (A1)). The ensuing classical correspondence is in line with Ehrenfest's theorem, though in a stronger

form: in case in which the initial wavefunction is a superposition of Gaussians (A9) with different parameters $q_{0}$ and $p_{0}$, Ehrenfest's theorem would then apply for each individual propagating wavepacket.

We will be interested in cases in which the time-dependent part of the potential, $V(t)$ is periodic. The stability properties of the general solutions of A3 - Hill's equation are well-known [33] by resorting to Floquet theory. For the present purpose of this work, it will suffice to restrict the discussion to the simplest non-trivial case, namely when the time-dependence takes the form

$$
V(t)=v-\kappa \cos (2 \omega t)
$$

Then Eq. A3 becomes a Mathieu equation and the guiding trajectory $q(t)$ is given in terms of real even ("cosine") and odd ("sine") Mathieu functions. It is well-known [34] that for a given $\kappa$, the solutions are bounded and periodic only for specific "characteristic values" of $v(\kappa)$.

[1] L. S. Schulman 1996, Techniques and Applications of Path Integration (Wiley, New York).

[2] P. R. Holland, 1995 The Quantum Theory of Motion (Cambridge Univ. Press, Cambridge).

[3] S. Wimberger 2014 Nonlinear Dynamics and Quantum Chaos (Springer, Heidelberg).

[4] S. Freund, R. Ubert, E. Flöthmann, K. Welge, D. M. Wang, and J. B. Delos 2002, Phys. Rev. A 65, 053408; C. Bordas, F. Lépine, C. Nicole, and M. J. J. Vrakking 2003 Phys. Rev. A 68, 012709. A. Matzkin, M. Raoult and D. Gauyacq 2003 Phys. Rev. A 68 , 061401(R); 
M. Lebental, N. Djellali, C. Arnaud, J.-S. Lauret, J. Zyss, R. Dubertrand, C. Schmit, and E. Bogomolny 2007 Phys. Rev. A 76, 023830; J. D. Wright, J. M. DiSciacca, J. M. Lambert, and T. J. Morgan 2010, Phys.Rev. A 81 , 063409 (2010); W. Gao, H. F. Yang, H. Cheng, X. J. Liu, and H. P. Liu 2012 Phys. Rev. A 86, 012517.

[5] Y. Nogami, F.M. Toyama, W. van Dijk 2000, Phys. Lett. A 270, 279; J. Acacio de Barros, G. Oliveira-Neto, and T.B. Vale 2005 Phys. Lett A 336324 A. Matzkin 2005, Phys. Lett. A 345, 31; X. Oriols 2007 Phys. Rev. Lett. 98, 066803; X. Y. Lai, Qing-yu Cai and M. S. Zhan 2009 New J. Phys. 11, 113035; S. Mousavi 2010 J. Phys. A: Math. Theor. 43 035304;

[6] R. E. Wyatt 2005 Quantum Dynamics with Trajectories (Springer, New York).

[7] A. Matzkin and V. Nurock 2008, Studies in Hist. and Phil. of Science B 39, 17; A. Matzkin 2009, Found. Phys. 39, 903.

[8] Y. Aharonov, D. Z. Albert, and L. Vaidman, Phys. Rev. Lett. 60, 1351 (1988).

[9] A. G. Kofman, S. Ashhab and F. Nori, Phys. Rep. 520, 43 (2012).

[10] J. Dressel, M. Malik, F. M. Miatto, A. N. Jordan, and R. W. Boyd 2014 Rev. Mod. Phys. 86, 307.

[11] A. Hosoya and Y.Shikano 2010 J. Phys. A 43385307.

[12] C. R. Leavens 2005, Found. Phys. 35, 469.

[13] H. M. Wiseman 2007, New J. Phys. 9165.

[14] B. J. Hiley 2012, J. Phys.: Conf. Ser. 361012014.

[15] F. L. Traversa, G. Albareda, M. Di Ventra, and X. Oriols 2013, Phys. Rev. A 87052124.

[16] S. Kocsis, B. Braverman, S. Ravets, M. J. Stevens, R. P. Mirin, L. K. Shalm and A. M. Steinberg 2011, Science 332, 6034, 1170.

[17] A. Matzkin 2012, Phys. Rev. Lett. 109, 150407.

[18] T. Mori and I. Tsutsui 2014, arXiv:1412.0916r1.

[19] D. Leibfried, R. Blatt, C. Monroe, and D. Wineland 2003, Rev. Mod. Phys. 75, 281

[20] V. V. Dodonov, M. A. Marchiolli, Ya. A. Korennoy, V. I. Man'ko, and Y. A. Moukhin 1998, Phys. Rev. A 58, 4087

[21] S. P. Kim and S. W. Kim 1995, Phys. Rev. D 514254.

[22] A. K. Pan and A. Matzkin 2012, Phys. Rev. A 85, 022122

[23] R. Jozsa 2007 Phys. Rev. A 76, 044103

[24] See the Supplemental Material of Ref. [17]. 
[25] S. V. Lawande and A. K. Dhara 1983, Phys. Lett. A 99353.

[26] L. de Broglie 1928, in Electrons et Photons (Gauthier-Villars, Paris), p. 105; D. Bohm 1952, Phys. Rev. 85, 166.

[27] B. J. Hiley and R. E. Callaghan 2012, Found. Phys. 42, 192.

[28] B. J. Hiley and R. E. Callaghan 2006, Phys. Scr. 74, 336.

[29] C. F. Lo 1993, Phys. Rev. A 47, 115.

[30] H. R. Lewis and W. B. Riesenfeld 1969 J. Math. Phys. 10, 1458.

[31] D. Schuch and M. Moshinsky 2006, Phys. Rev. A 73, 062111.

[32] J. R. Ray and J. L. Reid, J. Math. Phys. 22, 91 (1981); A. Matzkin 2001, J. Phys. A 34, 7833; F Haas 2002 J. Phys. A 35 2925; M Fernndez Guasti and H Moya-Cessa 2003 J. Phys. A 36 2069.

[33] A. H. Nayfeh and D. T. Mook 1995, Nonlinear oscillations (Wiley, New York).

[34] M. Abramowitz and I. A. Stegun (Eds) 1964, Handbook of Mathematical Functions (National Bureau of Standards, Washigton DC), Chap. 20. 